\title{
Le patch clamp: une nouvelle façon de voir les canaux ioniques
}

La technique du patch-clamp consiste à enregistrer les sauts de courant survenant lorsque, à voltage constant, l'état d'ouverture d'un canal ionique isolé se modifie. C'est grâce à cette approche qu'a pu être précisé l'essentiel des paramètres fonctionnels et cinétiques des canaux ioniques dont dépendent les propriétés électriques des cellules. Les interactions entre l'activité de ces canaux et l'ensemble du métabolisme biochimique des cellules sont également maintenant mieux connues grâce aux analyses en " patch-clamp ".

\section{Rémy Sauvé \\ Professeur agrégé, université de Montréal}

\section{RÉFÉRENCES}

1. Laüger $P$, Neumke B. Theoretical analysis of ion conductance in lipid bilayer membranes. In : Eisenman G, ed. Membrane Lipid Bilayers and Antibiotics. New York: Marcel Dekker, 1973 : 2-59.

2. Colquhoun D, Hawkes AG. On the stochastic properties of single ion channels. Proc $R$ Soc Lond (Biol) 1981; 211 : 205-35.

3. Hladky SB, Haydon DA. Ion transfer across lipid membranes in the presence of gramicidin A. Biochim Biophys Acta $1972 ; 274$ : 294-312.

\section{ADRESSE ET TIRÉS À PART}

R. Sauvé : groupe de recherche en transport membranaire, département de physiologie, université de Montréal, Montréal, Québec

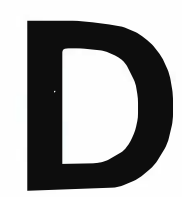

e nombreux phénomènes cellulaires tels que l'excitabilité, la contractibilité, la sécrétion, le transport de sucres et d'acides aminés, sont intimement liés aux propriétés électriques des cellules. Ces propriétés relèvent en grande partie de la perméabilité sélective de la membrane cellulaire aux ions. Cette membrane étant toutefois formée d'une double couche lipidique, constitue une barrière importante au passage des substances chargées. En effet, l'énergie requise pour amener un ion d'un milieu aqueux à un milieu lipidique est à ce point élevée que le passage transmembranaire des ions devient virtuellement impossible [1]. La membrane cellulaire contient toutefois certaines protéines dont la fonction est de catalyser le passage des ions, principalement le $\mathrm{K}^{+}$, le $\mathrm{Na}^{+}$, le $\mathrm{Cl}^{-}$et le $\mathrm{Ca}^{++}$. En fait, il existe trois catégories bien distinctes de complexes protéiques qui sont responsables de la perméabilité ionique des membranes biologiques. Dans un premier cas, il s'agit de pom- pes qui utilisent l'ATP cellulaire afin de maintenir l'intégrité ionique du milieu intracellulaire. On compte parmi ces protéines la $\mathrm{Na}^{+}-\mathrm{K}^{+}$ATPase et les $\mathrm{Ca}^{++}$ ATPases. D'autres protéines permettent le passage des ions grâce à des systèmes d'échanges ou de cotransport. On retrouve dans cette catégorie les échangeurs $\mathrm{Na}^{+}-\mathrm{Ca}^{++}$ou $\mathrm{Na}^{+}-\mathrm{H}^{+}$, de même que les systèmes de cotransport de sucres et d'acides aminés. Enfin, certaines molécules forment des canaux transmembranaires ou pores à l'intérieur desquels les ions peuvent diffuser passivement. Le flux ionique est, dans ce cas, dépendant du gradient électrochimique qui prévaut pour chacun des ions diffusibles. Les développements récents en électrophysiologie, en particulier la mise au point au début des années 80 de la technique du patch clamp, rendent actuellement possible l'étude des canaux ioniques au niveau de canaux individuels. Ces études ont permis, entre autres, de mettre en évidence à la fois la diversité et l'universalité des canaux ioniques 
comme structure de transport transmembranaire et de déterminer comment certains mécanismes de contrôle qui régissent l'activité des canaux ioniques sont reliés à l'ensemble des processus biochimiques qui ont cours à l'intérieur de la cellule.

\section{Les canaux ioniques}

Les canaux ioniques sont caractérisés par cette propriété particulière de fluctuer entre différents états moléculaires qui permettent (ouverts) ou ne permettent pas (fermés) le passage des ions. Ce processus de fluctuations est essen- tiellement stochastique, ce qui signifie que les règles qui régissent le passage d'un état à un autre sont de nature probabiliste [2]. Dans ce contexte, on ne peut en effet prévoir l'état futur d'un canal à partir de son état passé mais on peut seulement établir la probabilité qu'un tel état se produise. L'importance des canaux ioniques vient en grande partie du fait qu'un canal dans un état dit ouvert peut à lui seul catalyser le passage de $10^{6}$ à $10^{7}$ ions $/ \mathrm{sec}$, ce qui est $10^{3}-10^{4}$ fois plus rapide que tout système de pompe ou de transporteur connu. Les flux ioniques engendrés par l'ouverture d'un canal sont donc importants et nécessitent des mécanismes de contrôle d'une grande efficacité.

\section{Le patch clamp, principes de base}

Ce sont les propriétés de flux ioniques élevés ( $10^{6}$ ions/sec) et de transitions rapides entre états de conduction et non-conduction qui conferent à l'étude électrophysiologique des canaux ioniques un caractère unique. Ces deux caractéristiques permettent en effet d'effectuer des mesures électriques sur des canaux individuels. Les concepts fondamentaux à la base

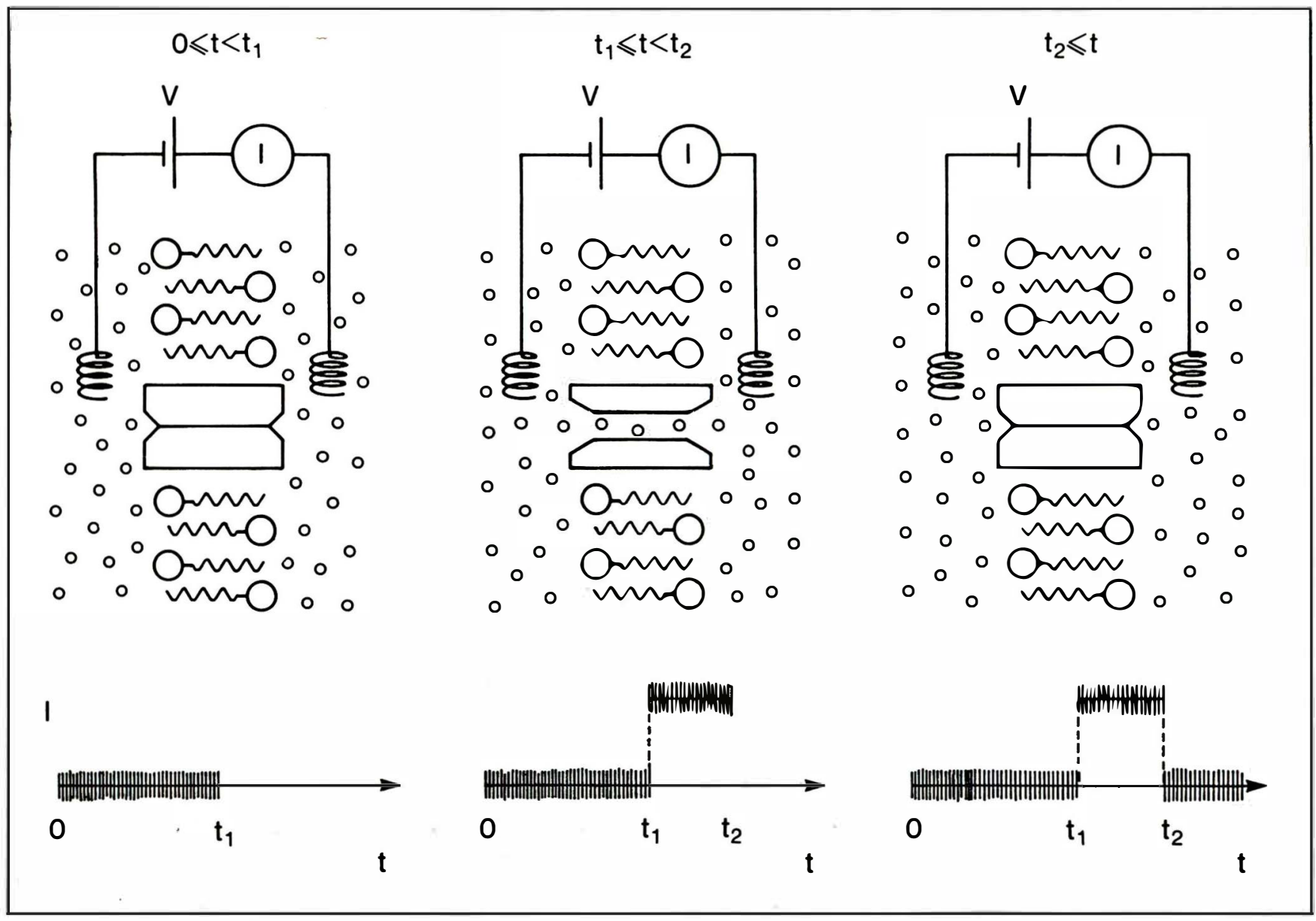

Figure 1. Représentation schématique de la corrélation existant entre la mesure de sauts de courant d'une part et les fluctuations d'un canal entre états fermés ot ouverts d'autre part. $A t<t_{1}$, le canal est dans un état fermé, ne permettant pas le passage des ions. Le courant mesuré en condition de voltage constant est alors nul. A $t_{1}$, une transition de l'état fermé vers l'état ouvert se produit. Si le canal demeure dans cet état ouvert pendant une période de temps $t_{2}-t_{1}$, un courant constant $d^{\prime}$ amplitude $\Delta i$ est alors mesuré durant cette période. A $t_{2}$, une nouvelle transition se produit de l'état ouvert vers l'état fermé. Le courant mesuré retourne alors à son niveau d'origine. Le résultat final apparaît donc comme un saut de courant d'amplitude constante dont la durée sera égale au temps passé par le canal dans l'état ouvert. 


\section{RÉFÉRENCES}

4. Neher E, Sakmann B. Single-channel currents recorded from membrane of denervated frog muscle fibres. Nature 1976 ; 260 : 794-802.

5. Hamill OP, Marty A, Neher E, Sakmann B, Sigworth FJ. Improved patch clamp technique for high-resolution current recording from cells and cell-free membrane patches. PYlugers Arch $1981 ; 391$ : 85-100.

6. Cooper K, Jakobson E, Wolynes P. The theory of ion transport through membrane channels. Prog Biophys Mol Biol 1985; 46 : 51-96.

7. Roux B, Sauvé R. A general solution to the time interval omission problem applied to single channel analysis. Biophys $J 1985 ; 48$ : 149-58

8. Colquhoun D, Sigworth FJ. Fitting and statistical analysis of single channel records. In Sakmann B, Neher E, eds. Single-Channel Recording. New York, London: Plenum Press, 1983 : 191-263.

9. Hille B. Ionic Channels of Excilable Membranes. Sunderlands: MA, Sinauer Associates IMC, 1984.

10. Guharay F, Sachs F. Stretch-activated single ion channel currents in tissue-cultured embryonic chick skeletal muscle. J. Physiol (Lond) 1984 ; 352 : 685-701.

11. Latorre R, Miller C. Conduction and selectivity in potassium channels. $J \mathrm{Membr}$ Biol $1983 ; 71: 11-30$

12. Karlin A. The anatomy of a receptor. Nourosci Comment 1983, $1: 111-23$.

13. Breitwieser GE, Szabo G. Uncoupling of cardiac muscarinic and B-adrenergic receptors from ion channels by a guanine nucleotide analogue. Nature 1985 ; 317 : 538-40.

14. Kurachi $Y$, Nakajima $T$, Sugimoto $T$. On the mechanism of activation of muscarinic $\mathrm{K}^{+}$channels by adenosine in isolated atrial cells ; involvement of GTP-binding proteins. Pflugers Arch 1986 ; 407 : 264-74.

15. Yatani A, Codina J, Brown AM, Birnbaumer L. Direct activation of mammalian atrial muscarinic potassium channels by GTP regulatory protein $G_{K}$. Sience 1987 ; 235 : 207-10.

16. Rasmussen H, Barrett P. Calcium messenger system : an integrated view. Physiol Reo 1984 ; 64 : 938-84.

17. Siegelbaum SA, Belardetti F, Camaro JS, Shuster MJ. Modulation of the serotoninesensitive potassium channel in Aplysia sensory neurone cell body and growth cone. $J$ Exp Biol $1986 ; 124: 287-306$ de ce type de mesures ont été en grande partie élaborés lors d'études sur des membranes artificielles à partir de molécules dites " formeurs de pore ", dont la plus connue est certes la gramicidine [3]. Un des points majeurs de ces études a été de démontrer que l'ouverture d'un canal se traduisait en condition de voltage imposé, par un saut de courant d'amplitude constante et de durée variable. L'idée fondamentale à la base de l'étude des canaux ioniques tient donc au fait qu'il existe une corrélation directe entre la mesure de sauts de courant d'une part et les fluctuations d'un canal entre différents états ouvertsfermés, d'autre part (figure 1, p. 539). Le patch clamp est l'approche expérimentale qui permet actuellement de mesurer les fluctuations de courant engendrées par des canaux ioniques in situ, c'est-à-dire directement à partir de membranes biologiques. Cette technique fut introduite d'abord par Neher et Sackmann en 1976 [4] et décrite dans sa forme améliorée en 1981 par Hamill et al. [5]. Cette méthode permet d'isoler électriquement une infime partie de la membrane cellulaire en pressant sur la surface externe d'une cellule une pipette de verre dont la pointe, de l'ordre de $1 \mu \mathrm{m}$, a été préalablement polie par chauffage. On obtient ainsi des résistances de contact entre le verre et la surface membranaire supérieures à $10^{10} \Omega$, ce qui rend possible la mesure des fluctuations de courant inférieures à $0,2 \mathrm{pA}$ à des fréquences de $1 \mathrm{kHz}$ ou plus (figure 2).

L'application de cette technique peut se faire selon diverses configurations (figure 3). Dans la configuration dite cell attached, la pipette de patch demeure sur la surface externe de la cellule entière. Cette approche est particulièrement utile pour l'étude des

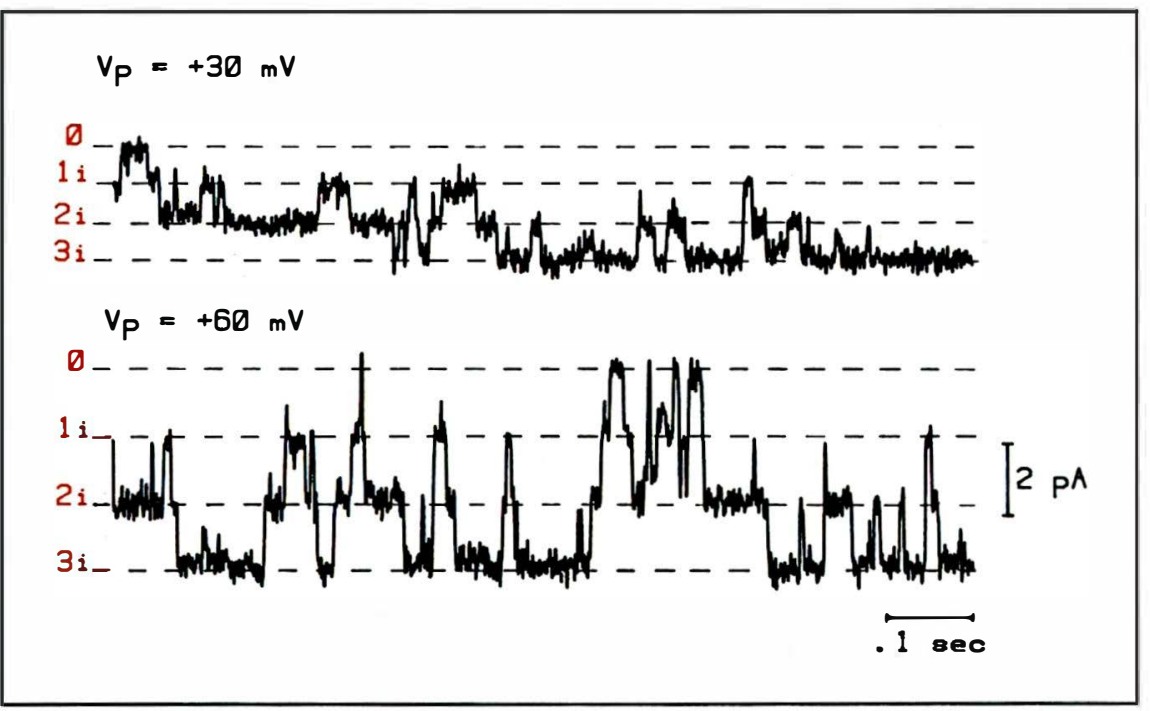

Figure 2. Exemple d'enregistrements de canaux sélectifs pour le $\mathrm{K}^{+}$ obtenus sur des cellules endothéliales d'aorte bovine par la méthode du patch clamp à différents voltages transmembranaires. Les deux principales caractéristiques associées à l'activité électrique de canaux ioniques y sont illustrées. Premièrement, les sauts de courant mesurés sont tous d'égale amplitude. Cette caractéristique conduit, pour un système comprenant plusieurs canaux, à des niveaux de courant dont les valeurs correspondent à des multiples entiers du niveau de courant associé à un seul canal ouvert. Deuxièmement, la durée des intervalles pour chacun des niveaux se distribue de façon statistique. Dans ce contexte, on ne peut prévoir quand se produiront exactement les transitions entre niveaux différents mais seulement la probabilité que de telles transitions se produisent. (Enregistrements obtenus en configuration "cell attached" avec une pipette contenant $200 \mathrm{mM} \mathrm{KCl}, 0,5 \mathrm{mM} \mathrm{MgSO}$, $1 \mu \mathrm{M} \mathrm{CaCl}{ }_{2}$ à $\mathrm{pH}$ 7,3. Signal filtré à $600 \mathrm{~Hz}$.) 


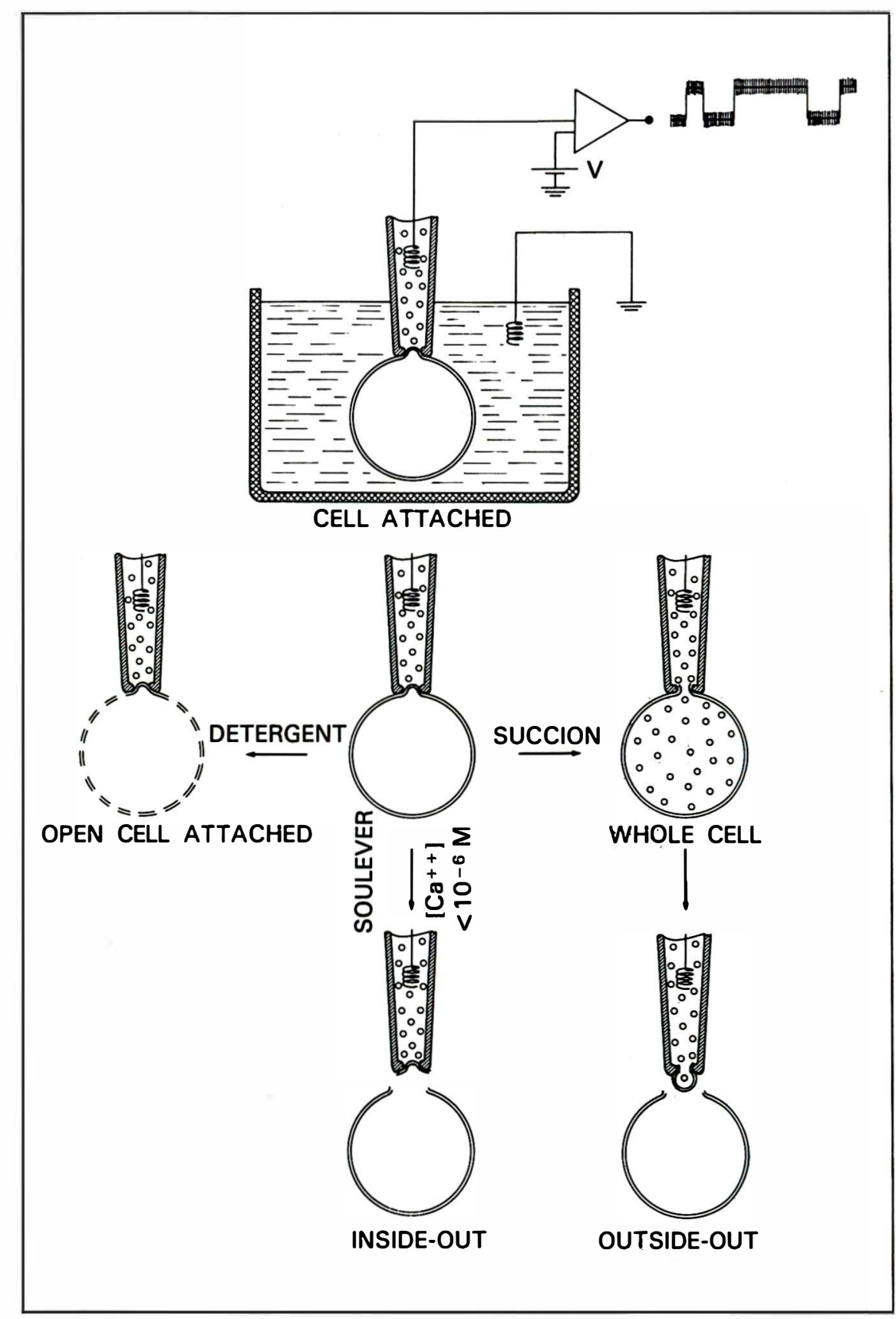

Figure 3. Représentation schématique de la méthode du patch clamp appliquée selon différentes configurations. Dans la configuration de base dite "cell attached", la pipette de patch est déposée sur la cellule et la membrane cellulaire aspirée légèrement à l'intérieur de l'électrode. II en résulte alors un contact électrique supérieur à $1010 \Omega$. La rupture de la membrane sous la pipette de patch mène à la configuration " whole cell ", à partir de laquelle la variante " outside-out" peut être obtenue. En soulevant la pipette de patch en configuration " cell attached", il est possible d'extraire, à faible concentration de calcium dans le bain $(<1 \mu \mathrm{M})$, un morceau de membrane dont la surface interne est alors mise en contact avec le milieu ionique du bain. Cette configuration dite "inside-out " peut aussi être recréée en perméabilisant la membrane cellulaire à l'aide de détergent tout en conservant la configuration "cell attached ". Cette variante se nomme alors " open cell attached".

$m / s n^{\circ} 9$ vol. 3, noombre 87 processus d'activation de canaux ioniques via des messagers intracellulaires tels le calcium ou l'AMP cyclique. A partir de la configuration cell attached, il est aussi possible de détruire, soit par succion, soit en appliquant un voltage élevé, la membrane sous la pipette de patch tout en conservant le contact entre la membrane et le verre. Dans ces conditions, la solution de remplissage de la pipette communique avec le milieu cytoplasmique et la pipette de patch peut être utilisée pour des expériences de voltage imposé sur toute la cellule. Cette variante dite whole cell permet aussi d'injecter dans la cellule certaines substances telles que des enzymes et des nucléotides dont l'action est susceptible de moduler l'activité électrique des canaux ioniques. A partir de cette configuration, on peut retirer la pipette de la cellule et obtenir ainsi, dans certaines conditions, un morceau isolé de membrane dont la surface externe est exposée vers l'extérieur. Cette variante dite outsideout est de préférence employée lors d'expériences où l'environnement de la surface externe nécessite d'être modifié. Enfin, si on soulève la pipette de patch à partir de la configuration cell attached, il est possible d'obtenir un infime morceau de membrane isolée dont la surface cytoplasmique est alors mise en contact avec les divers milieux ioniques du bain. Une telle configuration appelée insideout s'est avérée particulièrement fructueuse lors de l'étude de canaux ioniques activés par certains agents chimiques intracellulaires tels le calcium. Notons enfin que des conditions expérimentales, equivalentes à la configuration inside-out, ont été aussi obtenues en perméabilisant la cellule à l'aide de détergents (par ex. : saponine) afin d'obtenir la configuration open cell attached.

\section{Techniques d'analyse}

L'observation des sauts de courant, provenant de l'ouverture et de la fermeture d'un seul canal, nous permet deux niveaux dis- 
tincts d'analyse. Tout d'abord, l'analyse de la sélectivité ionique et de la conductance des canaux. L'amplitude des sauts est un indice de la facilité avec laquelle certains ions peuvent diffuser au travers du pore. A partir de la mesure de la conductance du canal en fonction du voltage et de la concentration d'ions plus ou moins diffusibles, il est en fait possible de déterminer le degré de sélectivité ionique du canal, l'affinité et le nombre minimum de sites où les ions peuvent fortement interagir avec le pore. Cette analyse repose sur une représentation physico-mathématique qui associe au canal une série de barrières d'énergie que les ions doivent surmonter lors du processus de diffusion [6]. Ces études s'adressent essentiellement à la fonction de transport du canal en indiquant quels ions peuvent diffuser et à quelle vitesse. Les canaux sont alors classés selon leur sélectivité, par exemple canal potassique ou canal sodique, et selon leurs caractéristiques courant-voltage : courbes courantvoltage linéaires ou autres.

Le second niveau d'analyse associé à l'étude des sauts, se rapporte à la cinétique d'ouverture et de fermeture du pore. Comme il a déjà été mentionné, les sauts de courant proviennent de l'ouverture et de la fermeture des canaux ioniques. L'analyse cinétique du signal consiste à déterminer la probabilité globale d'ouverture du canal, de même que la distribution statistique des intervalles de temps d'ouverture et de fermeture. La probabilité globale d'ouverture d'un canal s'obtient en mesurant la fraction du temps total passée dans tous les états ouverts, c'est-à-dire conducteurs. Cette mesure est importante car elle traduit directement l'effet des mécanismes de contrôle cellulaire sur les canaux ioniques. La détermination de la probabilité d'ouverture permet aussi d'extrapoler, à partir de mesures obtenues sur des canaux individuels, le comportement global qui devrait prévaloir pour tiques sur une cellule entière. L'étude statistique de la distribution des temps de fermeture et d'ouverture permet d'élaborer des modèles cinétiques décrivant la relation entre les états ouverts et fermés. Cette analyse comporte toutefois des limites intrinsèques. Premièrement, la détermination des intervalles de temps ouvertfermé nécessite la détection de transitions entre niveaux de courant différents. Les intervalles de très courte durée $(<0,3 \mathrm{msec})$ sont généralement omis, principalement à cause de la fréquence finie à laquelle les signaux de patch clamp sont enregistrés. Cette omission a pour effet de modifier complètement la façon dont les intervalles ouverts ou fermés se distribuent statistiquement. Certains formalismes mathématiques permettent toutefois de tenir compte de ces omissions lors de l'analyse des résultats expérimentaux [7]. Deuxièmement, l'analyse de ces distributions statistiques se fait à partir de fonctions multiexponentielles [8]. Le nombre d'exponentielles nécessaires pour reproduire la distribution des temps d'ouverture ou de fermeture correspond théoriquement au nombre total d'états ouverts ou fermés. Toutefois, à cause du degré de précision limité dans la mesure de ces distributions, le nombre d'états calculés ne représente généralement que le nombre minimum d'états pouvant rendre compte des résultats expérimentaux. Dès lors, la description d'un modèle cinétique unique devient dans la très grande majorité des cas impossible.

La caractérisation d'un ensemble de paramètres tels que la conductance, la sélectivité, la probabilité d'ouverture et les taux de transitions, concourt à la description précise d'un canal ionique donné. Lorsque la caractérisation est acquise, il devient alors possible

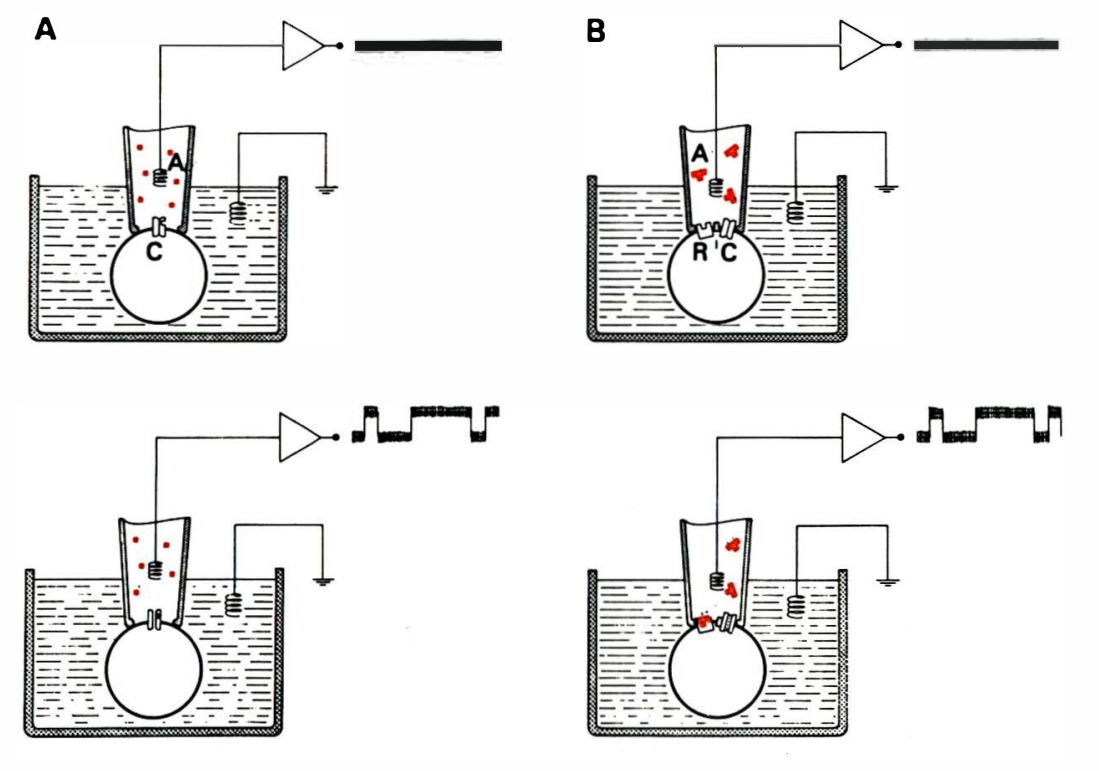

Figure 4. Contrôle biochimique des canaux par des mécanismes ne nécessitant pas de seconds messagers. A. le contrôle peut être direct s'il y a liaison entre l'agoniste (A) et la protéine membranaire assurant la fonction de canal (C). B. Ce contrôle peut toutefois être indirect si la stimulation d'un récepteur membranaire $(R)$ a pour effet $d^{\prime}$ activer une protéine intermédiaire capable de moduler l'activité électrique d'un canal. Dans tous ces cas, une action de I'agoniste sur le canal en configuration "cell attached " ou "inside-out" requerra la présence de l'agoniste dans la pipette de patch. Cette condition n'est toutefois pas nécessaire pour des mécanismes basés sur la présence de seconds messagers. 
de relier l'activité d'un type particulier de canal à l'ensemble des processus biochimiques régissant l'activité d'une cellule.

\section{Contrôle biochimique des canaux ioniques}

Le courant macroscopique moyen I provenant de $\mathrm{N}$ canaux ayant une probabilité d'ouverture $\mathrm{P}_{\text {。 }}$ est donné par le produit $\mathrm{I}=$ $\mathrm{NP}_{\mathrm{o}} \mathrm{i}$ où $\mathrm{i}$ est le courant au travers d'un seul canal. Le contrôle cellulaire d'un courant ionique peut donc en principe s'effectuer par une modification de chacun de ces paramètres. Sur la base de leurs mécanismes de régulation, les canaux ioniques peuvent être divisés en trois grandes catégories. Ceux dont l'activité est contrôlée par un facteur physique comme le voltage transmembranaire [9], la tension mécanique de la membrane [10], etc. : ceux dont le contrôle est assuré par un ou des agents chimiques comme le calcium, l'AMP cyclique ; et enfin ceux qui dépendent à la fois de facteurs physiques et d'agents chimiques. Certains canaux potassiques, dont la probabilité d'ouverture est fonction du calcium intracellulaire et du voltage, se situent dans cette dernière catégorie [11]. L'importance des canaux activés chimiquement provient en majeure partie de leur implication dans la réponse cellulaire à une variété de stimuli externes tels que les hormones ou les neutrotransmetteurs. Le couplage entre canaux ioniques et stimuli externes peut quant à lui s'effectuer selon divers mécanismes, certains nécessitant la présence de seconds messagers, d'autres non. La technique du patch clamp en configuration cell attached permet à cet égard de distinguer de façon non équivoque entre ces deux modes de fonctionnement. Dans un cas (couplage sans seconds messagers), l'activation des canaux sous la pipette de patch requerra la présence directe de l'agoniste dans l'électrode. On parlera alors de couplage direct ou intermédiaire entre stimuli et canaux (figure $4 A$ et $B$ ). Dans l'autre cas (couplage $m / s n^{\circ} 9$ vol. 3 , novembre 87 par l'intermédiaire de seconds messagers), l'activité des canaux pourra être modulée par la stimulation sélective de récepteurs localisés hors de la surface membranaire isolée par la pipette de patch (figure 5). La configuration cell attached assurant qu'il n'y a aucune interaction directe entre canaux et agoniste, une action de l'agoniste sur un canal devra nécessairement, dans ce contexte, impliquer la présence de seconds messagers intracellulaires.

Couplage sans seconds messagers. A la lumière des données qui existent aujourd'hui dans la littérature, on peut regrouper

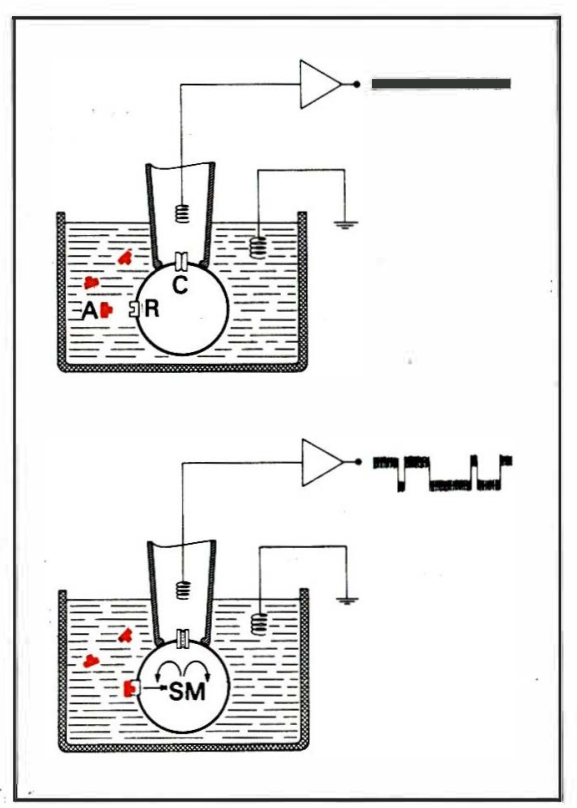

Figure 5. Application de la méthode du patch clamp en configuration " cell attached " à l'étude des mécanismes cellulaires reliant canaux ioniques (C) ot stimuli externes (A) via la production de seconds messagers (SM). L'approche expérimentale utilisée consiste à stimuler sélectivement par un agoniste (A) des récepteurs (R) localisés hors de la surface membranaire isolée par la pipette de patch. Si l'action de l'agoniste se répercute sur l'activité électrique des canaux sous la pipette, on peut alors conclure que le lien entre agoniste et canal nécessite la présence d'un second messager. La solution à l'intérieur de l'électrode de patch ne contenant pas d'agoniste, une interaction directe ne peut être dans ce cas invoquée. selon deux classes bien distinctes les mécanismes ne requérant aucun second messager.

Premièrement, le couplage direct : dans certains cas, le canal ionique possède les caractéristiques d'un récepteur membranaire. Dans ces conditions, la liaison d'un agoniste sur le canal produit un changement conformationnel qui mène au passage d'un état fermé à un état ouvert. C'est le cas en particulier des récepteurs nicotiniques à la jonction neuro-musculaire où les fonctions de canal et de récepteur sont assumées par une seule et même structure moléculaire [12]. Ce mode de fonctionnement est certes le plus direct car il ne nécessite aucun intermédiaire (figure 4A).

Deuxièmement, le couplage avec intermédiaires : des études récentes, en particulier sur le rôle des G-protéines dans l'activation de canaux potassiques après stimulation du récepteur muscarinique dans les cellules cardiaques, ont clairement démontré qu'il existe des mécanismes où la présence d'un agent intermédiaire est essentielle sans nécessairement impliquer la présence d'un second messager [13-15]. Le lien entre agoniste et canal ne peut être considéré comme étant direct dans ce cas, le canal ionique ne constituant plus une partie intégrale du récepteur membranaire. Le contrôle du canal serait transmis ici par une protéine intermédiaire, elle-même activée par la stimulation d'un récepteur membranaire (figure 4B). L'apport de la technique du patch clamp dans la configuration inside-out s'est avéré déterminant dans ce cas, puisqu'elle a permis de démontrer clairement l'implication de protéines intermédiaires sensibles au guanosine triphosphate (GTP) intracellulaire [14, 15].

Couplage avec seconds messagers. D'autres mécanismes de couplage entre stimuli externes et canaux ioniques nécessitent toutefois la production de seconds messagers. Des études électrophysiologiques basées sur la technique du patch clamp ont mis en évidence tout particulièrement le rôle de 


\section{RÉFÉRENCES}

18. Kameyama M, Hofmann F, Trautmein $W$. On the mechanism of $B$-adrenergic regulation of the $\mathrm{Ca}^{2+}$ channel in the guinea pig heart. Plugers Arch 1985 ; 405 : 285-93.

19. Hescheler J, Kameyama M, Trautmein $W$. On the mechanism of muscarinic inhibition of the cardiac current. Pflugers Arch 1986 ; $407: 182-9$

20. Marty A. $\mathrm{Ca}^{2+}$-dependent $\mathrm{K}^{+}$channels with large unitary conductance in chromaffin cell membranes. Nature 1981; 291 : 497-500.

21. Barrett JN, Magleby KL, Pallota BS. Properties of single calcium-activated potassium channels in cultured rat muscle. $J$ Physiol 1982 ; $331: 211-30$

22. Guggino SE, Suarez-Isla BA, Guggino W, Sacktor B. Forskolin and antidiuretic hormone stimulate $\mathrm{Ca}^{2+}$-activated $\mathrm{K}^{+}$channel in cultured kidney cells. Am J Physiol $1985 ; 249$ (Renal Fluid Electrolyte Physiol 18) : F448-55.

23. Sauvé R, Simoneau C, Monette R, Roy G. Single-channel analysis of the potassium permeability in HeLa cells : Evidence for a calcium-activated potassium channel of small unitary conductance. $J$ Membr Biol 1986 ; 92 : 269-82.

24. Suzuki K, Petersen $\mathrm{CCH}$, Petersen $\mathrm{OH}$ Hormonal activation of single $\mathrm{K}^{+}$channels via internal messenger in isolated pancreatic acinar cells. FEBS Lett 1985 ; 192 : 307-12.

25. Marty A, Evans MG, Tan YP, Trautmann A. Muscarinic response in rat lacrimal glands. $J$ Exp Biol 1986 ; 124 : 15-32.

26. Sauvé R, Simoneau C, Parent L, Monette R, Roy G. Oscillatory activation of calcium dependent potassium channels in $\mathrm{HeLa}$ cells induced by histamine $\mathrm{H}_{1}$ receptor stimulation : A single channel study. $J \mathrm{Mmbr}$ Biol 1987 ; 96 : 199-208.

27. Sterb $H$, Irvine RF, Berridge $M J$, Schultz I. Release of $\mathrm{Ca}^{+}+$from a non-mitochondrial intracellular store in pancreatic acinar cells by inositol-1, 4, 5-triphosphate. Nature 1983 ; 306 : $67-9$

28. Berridge MJ, Irvine RF. Inositol triphosphate : A novel second messenger in cellular signal transduction. Nature $1984 ; 312$ : 315-21.

29. Misler S, Falke LC, Gillis K, McDaniel ML. A metabolite-regulated potassium channel in rat pancreatic B cells. Proc Nall Acad Sci USA 1986, 83 : 7119-23.

30. Zimmerman AL, Baylor DA. Cyclic GMP-sensitive conductance of retinal rods consists of aqueous pores. Nature 1986 ; 321 : 70-2. deux messagers intracellulaires importants, à savoir l'AMP cyclique et le calcium.

Il est bien connu que l'activation de nombreux récepteurs membranaires se traduit par une augmentation intracellulaire en AMP cyclique via la stimulation de la protéine catalytique adénylate cyclase [16]. L'action de l'AMP cyclique se traduit à plusieurs niveaux. Or, il a été démontré chez l'Aplysie que l'activation par la sérotonine de récepteurs membranaires sur certains neurones amenait la fermeture d'un canal peu sensible au voltage et sélectif pour le potassium [17]. Cette action de la-sérotonine a été par la suite mise en relation avec une augmentation du niveau intracellulaire en AMP cyclique et la phosphorylation du canal via une protéine kinase [17]. Ce dernier point a d'ailleurs pu être confirmé lors d'expériences de patch clamp en configuration inside-out, dans lesquelles une protéine kinase reliée à l'AMP cyclique était directement ajoutée au milieu en contact avec la face cytoplasmique d'un morceau de membrane isolé [17]. Dans ce cas particulier, la phosphorylation du canal a eu pour conséquence la fermeture permanente du pore, produisant ainsi une variation du nombre total de canaux disponibles et non un changement uniforme de la probabilité d'ouverture des canaux. Cette action de l'AMP cyclique n'est toutefois pas universelle. D'autres travaux, portant entre autres sur des canaux sélectifs pour le calcium dans le muscle cardiaque, ont établi qu'une augmentation de l'AMP cyclique pouvait également provoquer l'activation de canaux ioniques [18, 19]. Il apparaît donc que les effets d'un même second messager peuvent être multiples, même si les modifications chimiques des canaux qui en sont à la base sont de même nature.

Plusieurs systèmes de récepteurs

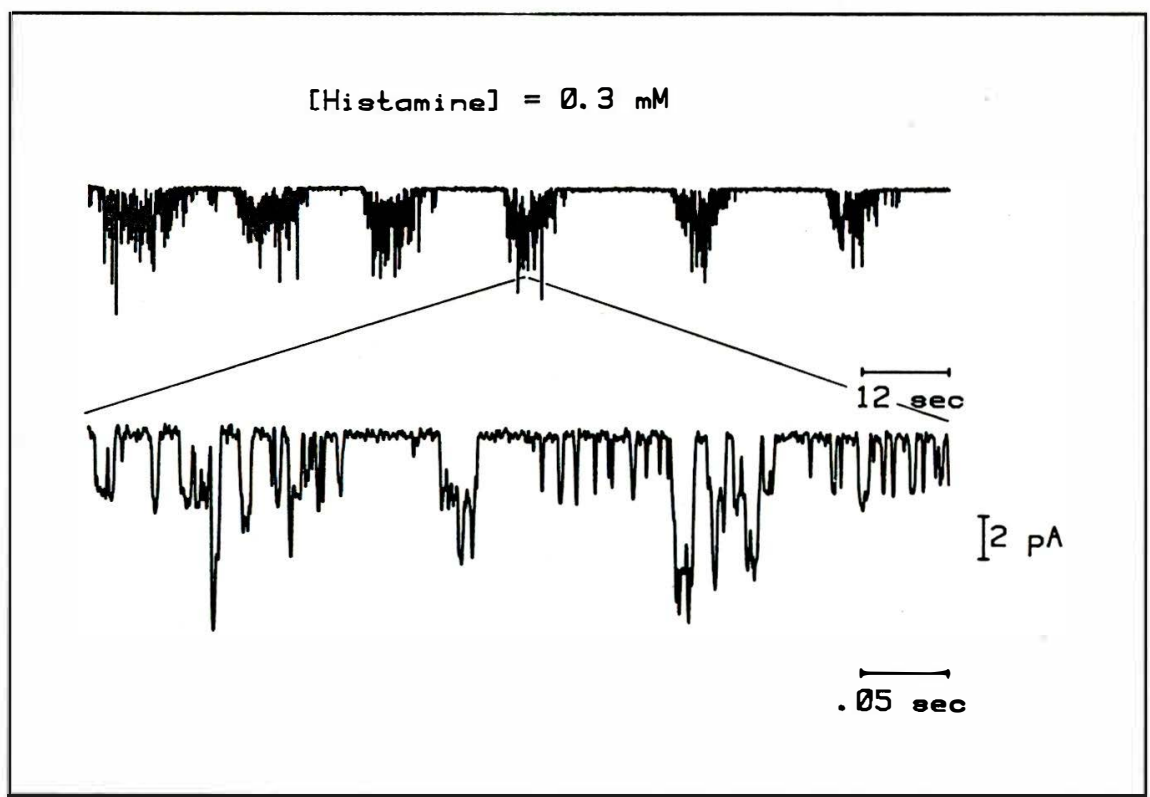

Figure 6. Exemple de réponse cyclique de canaux potassiques activés par lo calcium intracellulaire suite à la stimulation par l'histamine do réceptours $H_{1}$ sur los cellules HoLa. Chaque période d'activité est caractérisée par l'ouverture simultanée de plusieurs canaux. Ce processus d'activation peut être interprété en termes $d^{\prime} u n e$ variation cyclique de la concentration interne de calcium libre. La réponse de canaux potassiques activés par le calcium intracellulaire permet donc de mesurer des variations temporelles du niveau intracellulaire en calcium libre sur une seule cellule isolée. (Enregistrements obtenus en configuration "cell attached " avec une électrode contenant $200 \mathrm{mM}$ $\mathrm{KCl}, 0,5 \mathrm{mM} \mathrm{MgSO}_{4}, 1 \mu \mathrm{M} \mathrm{CaCl}$ à $\mathrm{pH}$ 7,3. Signal filtré à $500 \mathrm{~Hz}$.) 
membranaires utilisent le calcium comme messager intracellulaire [16]. Il est bien connu que la stimulation de récepteurs hormonaux amène dans de nombreux cas une augmentation transitoire de la concentration interne en calcium. Or, cette augmentation du calcium intracellulaire permet aussi de moduler l'activité électrique de certains canaux ioniques, particulièrement des canaux sélectifs pour les ions K. En effet, plusieurs études ont mis en évidence la présence quasi-universelle de canaux potassiques dont la probabilité d'ouverture est contrôlée par le calcium intracellulaire [11, 20-23]. Ici encore, la contribution de la méthode de patch clamp a été particulièrement significative. Le rôle du calcium comme agent activateur sans l'aide de cofacteurs a pu en effet être directement démontré grâce à des expériences effectuées dans la configuration inside-out où l'on modifiait la concentration en calcium du milieu en contact avec la face cytoplasmique de la membrane cellulaire. Cette action du calcium est aussi à l'origine du contrôle biochimique de certains canaux ioniques via l'activation de récepteurs membranaires dont la stimulation entraîne une augmentation de la concentration intracellulaire en calcium [24-26]. Le lien entre canaux ioniques et stimuli externes se traduit dans ce cas par une variation de la probabilité d'ouverture de tous les canaux activés par le calcium et non par un changement du nombre total de canaux activables. Cette corrélation entre activité électrique et calcium intracellulaire a aussi permis de démontrer, via le changement cyclique d'activité d'un canal potassique activé par le calcium intracellulaire, qu'il peut $y$ avoir des oscillations du niveau intracellulaire en calcium libre suite à la stimulation de récepteurs membranaires [26] (figure 6). Parmi les mécanismes moléculaires invoqués pour rendre compte de l'inter-relation, par l'intermédiaire du calcium, entre récepteurs membranaires et canaux ioniques, plusieurs travaux ont impliqué la production, à partir de dérivés phosphorylés du phosphatidylinositol, d'inositol triphosphate $\left(\mathrm{IP}_{3}\right)[27,28]$. L'IP 3 serait l'agent directement responsable de la libération de calcium [28]. Ce mécanisme a d'ailleurs été confirmé de façon précise en mesurant dans la configuration whole cell l'activité électrique de cellules de glandes exocrines en présence d'IP $_{3}$ dans la pipette de patch [25].

En conclusion, la description que nous venons de faire des divers mécanismes reliant récepteurs membranaires et canaux ioniques est loin d'être exhaustive. De nombreux travaux portant sur le rôle de l'ATP intracellulaire [29] ou du guanosine monophosphate (GMP) cyclique comme agents régulateurs [30] ont aussi été effectués en utilisant la méthode du patch clamp. Cette description représente toutefois un aperçu de la contribution de la méthode du patch clamp à l'étude du contrôle biochimique des canaux ioniques. De fait, par sa souplesse d'emploi et la puissance de sa résolution, le patch clamp a permis une intégration plus profonde de l'étude des canaux ioniques à l'ensemble des processus physiologiques au niveau cellulaire. Les canaux ne sont plus dans ce contexte de simples molécules qui assurent le passage passif des ions, mais bien des structures qui interagissent avec l'ensemble de la machinerie moléculaire de la cellule. Des développements prometteurs sont à prévoir à ce niveau car ils mèneront de toute évidence à une convergence des données aussi bien électrophysiologiques que biochimiques et génétiques. La contribution du patch clamp à cette synthèse de la physiologie cellulaire aura été et sera certes d'une importance capitale

\section{Remerciements}

Je tiens à remercier le Dr Lucie Parent pour sa lecture critique du manuscrit.

\section{Summary}

Ionic channels are proteins that form hydrophilic pores in membranes. Channels can be either in an open or closed state depending on their ability to allow ions to flow passively through them or not. Of major importance is the observation that the rate of ionic flux through an open channel ranges from $10^{6}$ to $10^{7}$ ions $/ \mathrm{sec}$, which is at least $10^{3}$ times higher than the fluxes expected from membrane carrier type systems. Ionic channels are also characterized by the stochastic nature of their behavior. As a consequence, the time spent by a channel in an open or closed state cannot be predicted per se, but only the probability that such an event occurs. The combined effects of large ionic fluxes coupled to fast random transitions among different open and closed states make possible the recording of current fluctuations coming from a single channel molecule. Since the development of the patch clamp technique by Neher and Sakmann (1976), it became possible to measure single channel events in biological membranes. One major contribution of this method concerns the study of the cellular mechanisms underlying the control of ionic channels. For instance, patch clamp studies have clearly established the role of intermediate proteins, such as $G$ proteins, as functional links between membrane receptors and ionic channels. Other studies, focused especially on mechanisms requiring second messengers, have shown that the control of ionic channels can be achieved through cyclic AMP or internal calcium level regulation. The patch clamp method has thus provided a unique approach to understand the contribution of ionic channels to the cell global physiology. 